\title{
P2Y12 antagonist ticagrelor inhibits the release of procoagulant extracellular vesicles from activated platelets
}

\author{
Aleksandra Gasecka ${ }^{1,2,3}$, Rienk Nieuwland ${ }^{2,3}$, Edwin van der $\mathrm{Pol}^{2,3,4}$, Najat Hajji ${ }^{1}$, \\ Agata Cwiek ${ }^{1}$, Kinga Pluta ${ }^{1}$, Michał Konwerski ${ }^{1}$, Krzysztof J. Filipiak ${ }^{1}$ \\ ${ }^{1} 1^{\text {st }}$ Chair and Department of Cardiology, Medical University of Warsaw, Poland \\ ${ }^{2}$ Laboratory of Experimental Clinical Chemistry, Academic Medical Center \\ of the University of Amsterdam, The Netherlands \\ ${ }^{3}$ Vesicle Observation Center, Academic Medical Center of the \\ University of Amsterdam, The Netherlands \\ ${ }^{4}$ Biomedical Engineering and Physics, Academic Medical Center of the \\ University of Amsterdam, The Netherlands
}

\begin{abstract}
Background: Activated platelets release platelet extracellular vesicles (PEVS). Adenosine diphosphate $(A D P)$ receptors $P 2 Y 1$ and $P 2 Y 12$ both play a role in platelet activation, The present hypothesis herein is that the inhibition of these receptors may affect the release of PEVs.

Methods: Platelet-rich plasma from 10 healthy subjects was incubated with saline, P2Y1 antagonist MRS2179 (100 $\mu M)$, P2Y12 antagonist ticagrelor $(1 \mu M)$, and a combination of both antagonists. Platelets were activated by $A D P(10 \mu M)$ under stirring conditions at $37^{\circ} \mathrm{C}$. Platelet reactivity was assessed by impedance aggregometry. Concentrations of PEVs- (positive for CD61 but negative for $P$-selectin and phosphatidylserine) and PEVs + (positive for all) were determined by a state-of-the-art flow cytometer. Procoagulant activity of PEVs was measured by a fibrin generation test.

Results: ADP-induced aggregation (57 \pm 13 area under curve \{AUC] units) was inhibited $73 \%$ by the P2Y1 antagonist, $86 \%$ by the P2Y12 antagonist, and 95\% when combined ( $p<0.001$ for all). The release of PEVs- $\left(2.9 \mathrm{E} \pm 0.8 \times 10^{8} / \mathrm{mL}\right)$ was inhibited $48 \%$ in the presence of both antagonists $(p=0.015)$, whereas antagonists alone were ineffective. The release of PEVs $+\left(2.4 \pm 1.6 \times 10^{7} / \mathrm{mL}\right)$ was unaffected by the P2Y1 antagonist, but was $62 \%$ inhibited by the P2Y12 antagonist $(p=0.035)$, and $72 \%$ by both antagonists $(p=0.022)$. PEV promoted coagulation in presence of tissue factor. Conclusions: Inhibition of $P 2 Y 1$ and $P 2 Y 12$ receptors reduces platelet aggregation and affects the release of distinct subpopulations of PEVs. Ticagrelor decreases the release of procoagulant PEVs from activated platelets, which may contribute to the observed clinical benefits in patients treated with ticagrelor. (Cardiol J 2019; 26, 6: 782-789)
\end{abstract}

Key words: extracellular vesicles, platelets, ADP receptors, P2Y12 antagonists, ticagrelor

\section{Introduction}

Platelet activation and aggregation in response to atherosclerotic plaque rupture is a key event in the pathogenesis of acute coronary syndrome (ACS) [1]. Platelet P2Y1 and P2Y12 receptors for adenosine diphosphate (ADP) contribute to ACS by initiating and amplifying platelet activation, respectively [2]. Whereas antagonists against the P2Y1 receptor are under development [3], antagonists against the P2Y12 receptor are clinically established to prevent recurrent cardiovascular events in patients with ACS [4].

Address for correspondence: Aleksandra Gasecka, MD, $1^{\text {st }}$ Chair and Department of Cardiology, Medical University of Warsaw, Independent Public Central Teaching Hospital, ul. Banacha 1a, 02-097 Warszawa, Poland, e-mail: aleksandra.gasecka@wum.edu.pl 


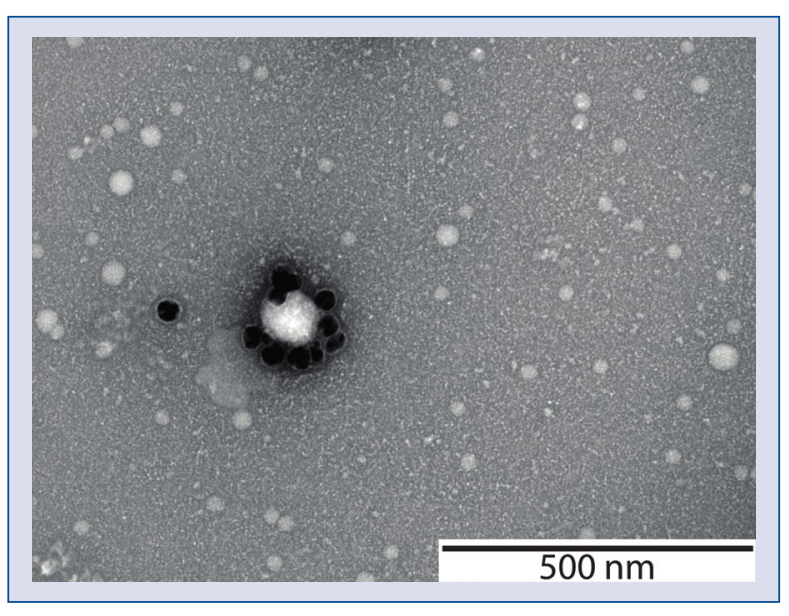

Figure 1. A transmission electron microscope image of platelet extracellular vesicles from human plasma. The vesicles were labelled with gold nanoparticles conjugated to CD61 antibodies. Image courtesy of Chi M. Hau, Vesicle Observation Center, Academic Medical Center, University of Amsterdam, the Netherlands.

In the course of ACS, activated platelets release fragments of their outer cell membrane to the bloodstream, called platelet-derived extracellular vesicles (PEVs) [5]. A transmission electron microscope image of PEVs from human plasma is shown in Figure 1. PEVs are nanoparticles surrounded by a phospholipid membrane which encloses cytoplasmic components such as proteins, lipids, metabolites and nucleic acids, they also contain platelet-derived proteins [5]. The expo- sure of glycoprotein (GP) IIb/IIIa, a receptor for fibrinogen (CD41/CD61) enables identification of $\mathrm{PEVs}$ released from platelets or megakaryocytes among other extracellular vesicles (EVs) present in blood. In turn, the exposure of GP IIb/IIIa along with P-selectin (CD62P) and phosphatidylserine (PS), which enables identification of PEVs released from activated platelets [6]. PEVs exposing P-selectin and PS are likely involved in inflammation and thrombosis [7-10]. P-selectin initiates binding of platelets and PEVs to monocytes via P-selectin glycoprotein ligand-1 (PSGL-1), leading to monocyte activation, cytokine release and exposure of tissue factor (TF) on monocytes [9]. PS along with other negatively charged phospholipids bind clotting factors in the presence of calcium ions, thereby propagating thrombin generation [10]. Hence, PEVs exposing P-selectin and PS may potentially contribute to the development and progression of atherosclerosis [11-13].

Although the potential applications of PEVs as biomarkers [14], risk predictors [15] and therapeutic targets [16] in cardiovascular disease are recognised, the mechanisms underlying the release of PEVs are unclear [17]. Because both P2Y1 and P2Y12 receptors regulate platelet activation, it was hypothesized that inhibition of these receptors may affect the release of PEVs.

\section{Methods}

The design of the experiments is shown in Figure 2.

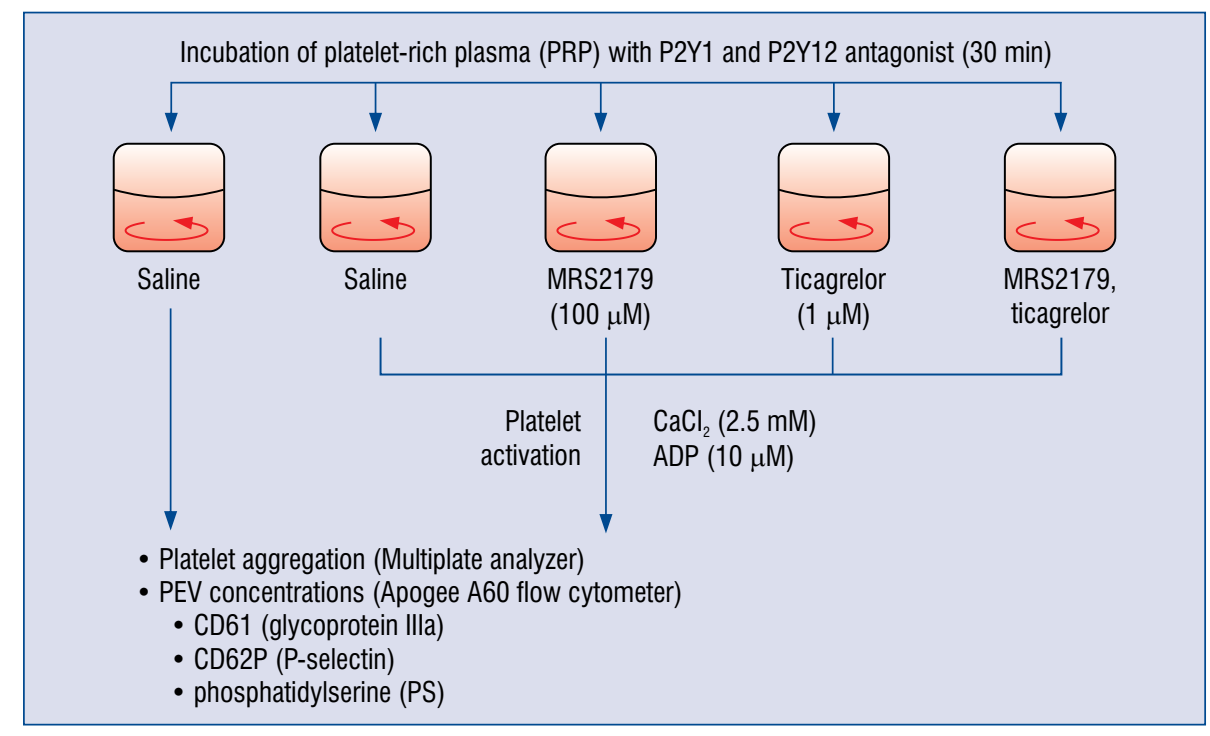

Figure 2. The design of the experiments on the role of the $\mathrm{P} 2 \mathrm{Y} 1$ and $\mathrm{P} 2 \mathrm{Y} 12$ receptors in the release of platelet extracellular vesicles. $\mathrm{CaCl}_{2}$ - calcium chloride; ADP - adenosine diphosphate; $\mathrm{CD}$ - cluster of differentiation; PEVs - platelet extracellular vesicles. 


\section{Materials}

Trisodium citrate blood collection tubes were obtained from Sarstedt (Germany). Needles were obtained from Vygon (Belgium). The P2Y1 antagonist MRS2179 was obtained from Tocris Biosciences (United Kingdom [UK]). The P2Y12 antagonist ticagrelor was obtained from Cayman Chemical (USA), ADP was from Bio/Data Corporation (USA), and acid citrate dextrose (ACD), phosphate-buffered saline (PBS), sodium citrate and calcium chloride $\left(\mathrm{CaCl}_{2}\right)$ were obtained from Sigma Aldrich (USA). The Multiplate analyser and reagents were from Roche Diagnostics (Germany), lactadherin-FITC from Hematologic Technologies (USA), CD61-APC from Dako (Denmark), and CD62P-violet and isotype controls from Becton Dickinson (USA). The membrane $(0.05 \mathrm{~nm})$ to filtrate the buffers was obtained from Merck Millipore (Germany). The Rosetta Calibration beads were obtained from Exometry (The Netherlands). Innovin (recombinant human TF) was obtained from Siemens (Germany) and anti-factor VIIa from Sanquin (The Netherlands).

\section{Blood collection and handling}

Blood samples were obtained from 10 healthy, drug-free donors after overnight fasting and giving informed consent, according to the recent guidelines [18]. Blood was collected using a 19 gauge needle and a plastic tube containing citrate (final concentration $0.109 \mathrm{~mol} / \mathrm{L}$ ). The tourniquet was removed promptly after the venepuncture. The first $2.5 \mathrm{~mL}$ of blood collected was discarded to avoid pre-activation of platelets. The tubes were kept in a vertical position for $15 \mathrm{~min}$. Platelet rich plasma (PRP) was prepared by centrifugation of blood for $180 \mathrm{~g}$ at $15 \mathrm{~min}$. Centrifugation was performed using Rotina $380 \mathrm{R}$ centrifuge equipped with a swing-out rotor and a radius of $155 \mathrm{~mm}$ (Hettich Zentrifugen, Germany) at room temperature with acceleration speed 1, without brake.

\section{Aggregometry}

$288 \mu \mathrm{L}$ of citrated PRP was added to $288 \mu \mathrm{L}$ saline and incubated for $30 \mathrm{~min}$ at $37^{\circ} \mathrm{C}$ with (i) saline, (ii) P2Y1 receptor antagonist MRS2179 (final concentration $100 \mu \mathrm{M}$ ), (iii) P2Y12 receptor antagonist ticagrelor (final concentration $1 \mu \mathrm{M}$ ), and (iv) a combination of both antagonists. Subsequently, the diluted PRP was re-calcified (final concentration $2.5 \mathrm{mM} \mathrm{CaCl}_{2}$ ) before addition of $\operatorname{ADP}(10 \mu \mathrm{M})$. Platelet aggregation was recorded for 6 min while stirring using Multiplate analyser (Roche Diagnostics, Germany).

\section{Flow cytometry}

Platelet-free supernatant was prepared by centrifugation of $600 \mu \mathrm{L}$ PRP with $120 \mu \mathrm{L} \mathrm{ACD}$ at $800 \mathrm{~g}$ for $20 \mathrm{~min}$. PEVs were measured in this plateletfree supernatant collected $30 \mathrm{~min}$ after the addition of ADP. Prior to labelling of the supernatant, antibodies were diluted in PBS and centrifuged at $18,890 \mathrm{~g}$ for $5 \mathrm{~min}$ to remove protein aggregates. To stain, $20 \mu \mathrm{L}$ of the supernatant was incubated with $2.5 \mu \mathrm{L}$ CD61-APC, $2.5 \mu \mathrm{L}$ CD62P-violet, $2.5 \mu \mathrm{L}$ lactadherin-FITC or isotype controls in the dark for $2 \mathrm{~h}$ at room temperature. The reaction was stopped by adding $200 \mu \mathrm{L}$ citrated PBS solution, adjusted to $\mathrm{pH} 7.4$ and filtered with a $0.05 \mu \mathrm{m}$ membrane prior to use [18]. PEVs released from platelets (positive for CD61 but negative for P-selectin and PS) are further referred to as PEVs-. PEVs released from activated platelets (positive for all) are referred to as PEVs + [6]. PEV concentrations were determined by A60 Micro flow cytometer (Apogee Flow Systems, UK) for $60 \mathrm{~s}$ at a flow rate of $3.01 \mu \mathrm{L} / \mathrm{min}$. Prior to measurement, the samples were diluted with PBS to 5,000 events per second to avoid swarm [19]. The applied voltages were $375 \mathrm{~V}, 520 \mathrm{~V}, 510 \mathrm{~V}$, and $500 \mathrm{~V}$ for the side scatter detector, FITC detector, APC detector, and violet detector, respectively. The gains were 1 . The trigger threshold was set on side scatter channel 10 . Rosetta Calibration was used to relate side scatter to the diameter of PEVs, assuming a PEV refractive index of 1.4 [20].

The fluorescent gates were based on the isotype controls. To analyse the concentrations of PEVs-, in the first step all PEVs exposing CD62P and PS were gated out, and PEVs exposing CD61 only were detected in the red channel (638-D Red). To analyse the concentrations of PEVs + , in the first step all plasma EVs exposing PS (binding to lactadherin) were detected in the green channel (488-Green). Out of all PEVs exposing PS, PEVs exposing additionally CD61 and CD62P were detected in the red channel (638-D Red) and blue channel (405-Blue). At the time of the experiment, no suitable beads to calibrate molecules of equivalent soluble fluorophores (MESF) were available for the APC and violet channel. Therefore, gates were set in arbitrary units (AU) with the following values: 4,153 AU for CD61-APC; 6,185 AU for CD62P-violet and 2,661 AU for lactadherin-FITC.

\section{Fibrin generation test}

The ability of PEVs to generate fibrin was measured in platelet-free plasma in the absence or presence of human recombinant $\mathrm{TF}$ (coagulation 


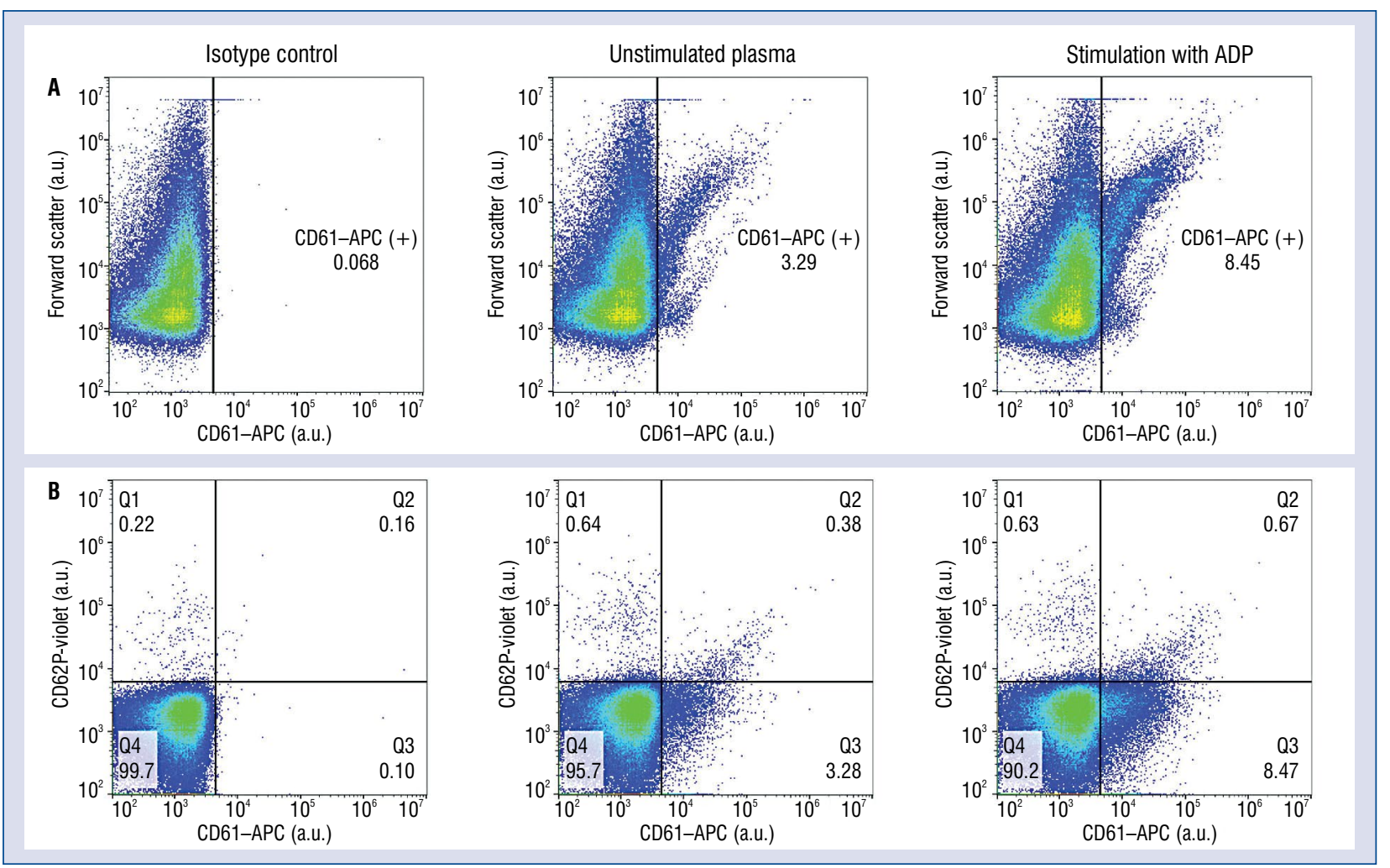

Figure 3. Representative images of platelet extracellular vesicles (PEVs) from adenosine diphosphate-activated platelets in plasma of a healthy donor measured by flow cytometry (A60-Micro, Apogee Flow Systems, Hertfordshire, UK); A. PEVs exposing CD61 but not CD62P and phosphatidylserine (PS) and labelled with anti-CD61-APC; B. PEVs exposing CD61, CD62P and PS and labelled with anti-CD61-APC, anti-CD62P-Violet and lactadherin-FITC, respectively. In the first step all plasma extracellular vesicles exposing PS (binding to lactadherin) were recorded (data not shown). Out of all PEVs exposing PS, PEVs exposing CD61 and CD62P were recorded (Q2); a.u. - arbitraty units.

factor VII), antibody against factor VIIa (anti-factor VIIa) and excess of lactadherin [21]. After preincubation for $5 \mathrm{~min}$ at $37^{\circ} \mathrm{C}$, clotting was initiated by addition of $\mathrm{CaCl}_{2}$. Fibrin formation over $1 \mathrm{~h}$ was determined by measuring the optical density $(\lambda=405 \mathrm{~nm})$ in duplicate on a spectrophotometer (SPECTRAmax microplate reader, Molecular Devices, USA) at $37^{\circ} \mathrm{C}$.

\section{Statistical analysis}

Kolmogorov-Smirnov test was used to test for Gaussian distribution. Data were analysed by paired Student's t-test. A Spearman rank correlation test was used to assess the correlation between aggregation results and concentration of PEVs. Statistical analysis was performed with the STATISTICA 12.0 (StatSoft, USA) and Prism 7.0 (GraphPad, USA) software. Flow cytometry data was analysed with FlowJo 9.2 (FlowJo, USA). Data are presented as mean \pm standard deviation. All p-values are given as two-sided values, with a p-value $<0.05$ considered significant.

\section{Results}

\section{Size distribution of PEVs}

Activation of platelets with ADP triggered platelet aggregation and the release of PEVs, compared to unstimulated platelets. The release of PEVs-and PEVs + in response to ADP is shown in Figure 3. Scatter-based size distribution of the total population of PEVs $\left(\mathrm{CD} 61^{+}\right)$confirmed that PEVs ranged from $150 \mathrm{~nm}$ to $1,000 \mathrm{~nm}$ in diameter, with the majority of PEVs being below $200 \mathrm{~nm}$. Size distribution of the total population of PEVs is shown in Supplementary Figure 1. Please note, that plasma contains (platelet) EVs down to $30 \mathrm{~nm}$, which are below the lower detection limit of flow cytometry [22].

The effects of $\mathrm{ADP}$ receptor antagonists on platelet aggregation and release of PEVs

Adenosine diphosphate-induced aggregation (57 \pm 13 area under curve [AUC] units) was $73 \%$ inhibited by the P2Y1 antagonist alone, $86 \%$ by 


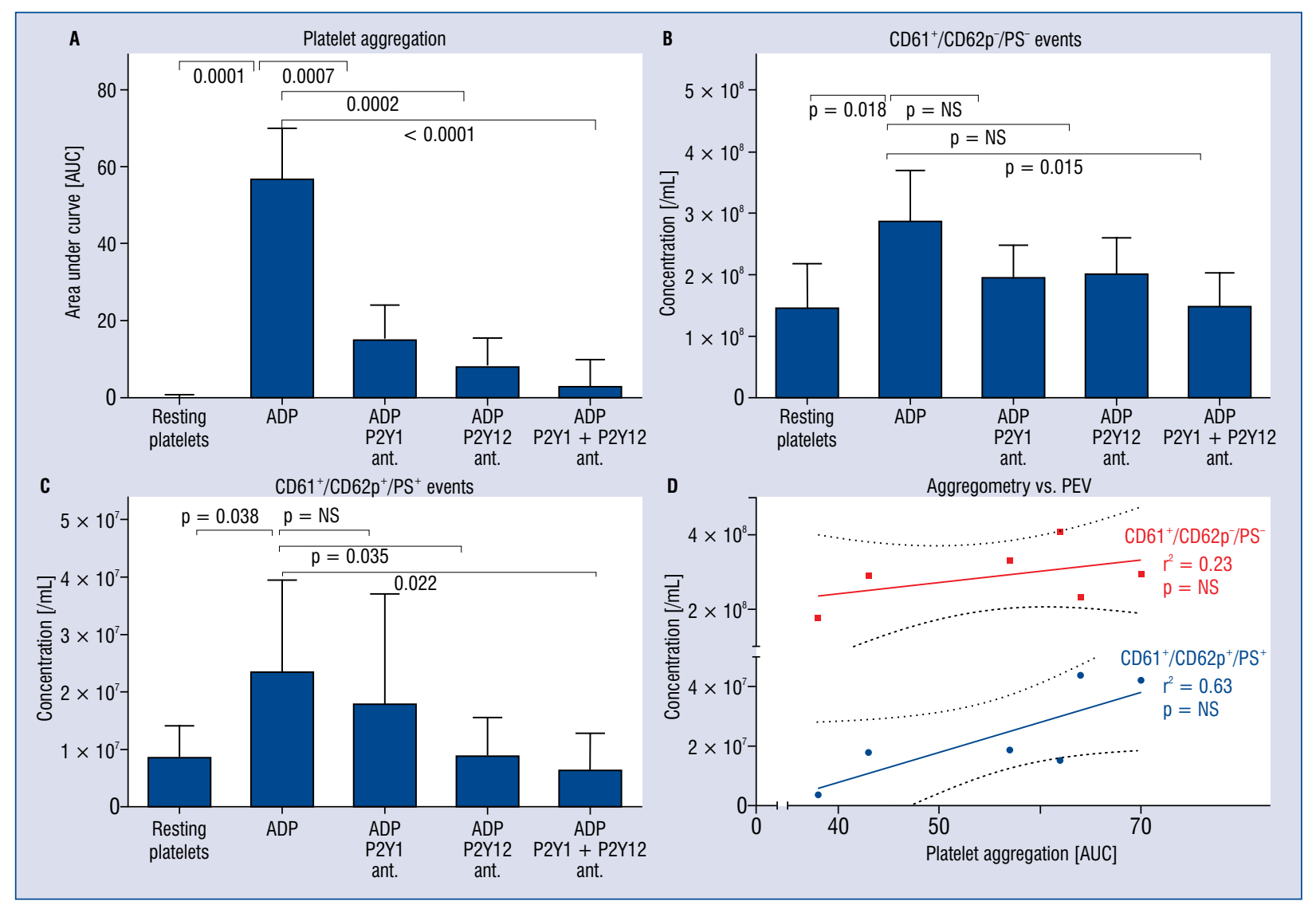

Figure 4. The effect of platelet pre-incubation with a P2Y1 antagonist, a P2Y12 antagonist and a combination of both antagonists on platelet aggregation (A) and the release of platelet extracellular vesicles (PEVs) exposing CD61 but not CD62p and phosphatidylserine (PS) (B) and PEVs exposing CD61, CD62p and PS (C) in response to adenosine diphosphate (ADP). Whereas platelet aggregation correlated weakly with the concentration of $\mathrm{CD} 61^{+} / \mathrm{CD} 6 \mathrm{P}^{-} / \mathrm{PS}^{-} \mathrm{PEVs}$, platelet aggregation correlated strongly with the concentration of $\mathrm{CD} 61^{+} / \mathrm{CD} 62 \mathrm{P}^{+} / \mathrm{PS}^{+} \mathrm{PEVs}$ (D).

the P2Y12 antagonist alone, and 95\% when both antagonists were combined ( $\mathrm{p}<0.001$ for all; Fig. 4A). The release of PEVs- $\left(2.9 \pm 0.8 \times 10^{8}\right.$ events $/ \mathrm{mL}$ ) was unaffected by pre-incubation with the P2Y1 antagonist or by the P2Y12 antagonist alone, but decreased by $50 \%$ in presence of both antagonists $(\mathrm{p}=0.015$; Fig. 4B). In contrast, the release of PEVs $+\left(2.4 \pm 1.6 \times 10^{7}\right.$ events $\left./ \mathrm{mL}\right)$ was unaffected by the P2Y1 antagonist alone, but was decreased $62 \%$ by the P2Y12 antagonist alone $(\mathrm{p}=0.035)$, and $72 \%$ in the presence of both antagonists ( $\mathrm{p}=0.022$; Fig. $4 \mathrm{C})$. Whereas the extent of platelet aggregation weakly correlated with the concentration of PEVs- $\left(\mathrm{r}^{2}=0.2\right)$, the extent of platelet aggregation strongly correlated with the release of PEVs $+\left(r^{2}=0.7\right.$, Fig. 4D).

\section{Procoagulant activity of PEVs}

Platelet-free plasma containing PS-exposing EVs from ADP-activated platelets did not clot up to
$1 \mathrm{~h}$ after re-calcification. When human recombinant $\mathrm{TF}$ was added, clotting was initiated. The magnitude of clotting was not affected by the presence or absence of P2Y1 and P2Y12 antagonists (data not shown). Clotting was abolished both by covering the PS by an excess of lactadherin and by inhibiting TF activity with anti-factor VIIa. The proposed procoagulant activity of $\mathrm{PEVs}$ measured with fibrin generation test is shown in Figure 5.

\section{Discussion}

P2Y1 and P2Y12 receptors differentially regulate platelet aggregation and the release of PEVs. Inhibition of either $\mathrm{P} 2 \mathrm{Y} 1$ or $\mathrm{P} 2 \mathrm{Y} 12$ receptor is sufficient to inhibit ADP-induced platelet aggregation [23], which is consistent with the clinical observation that P2Y12 antagonists reduce the incidence of thrombosis in patients after ACS [4]. Further, inhibition of the $\mathrm{P} 2 \mathrm{Y} 1$ and $\mathrm{P} 2 \mathrm{Y} 12$ receptors affects 


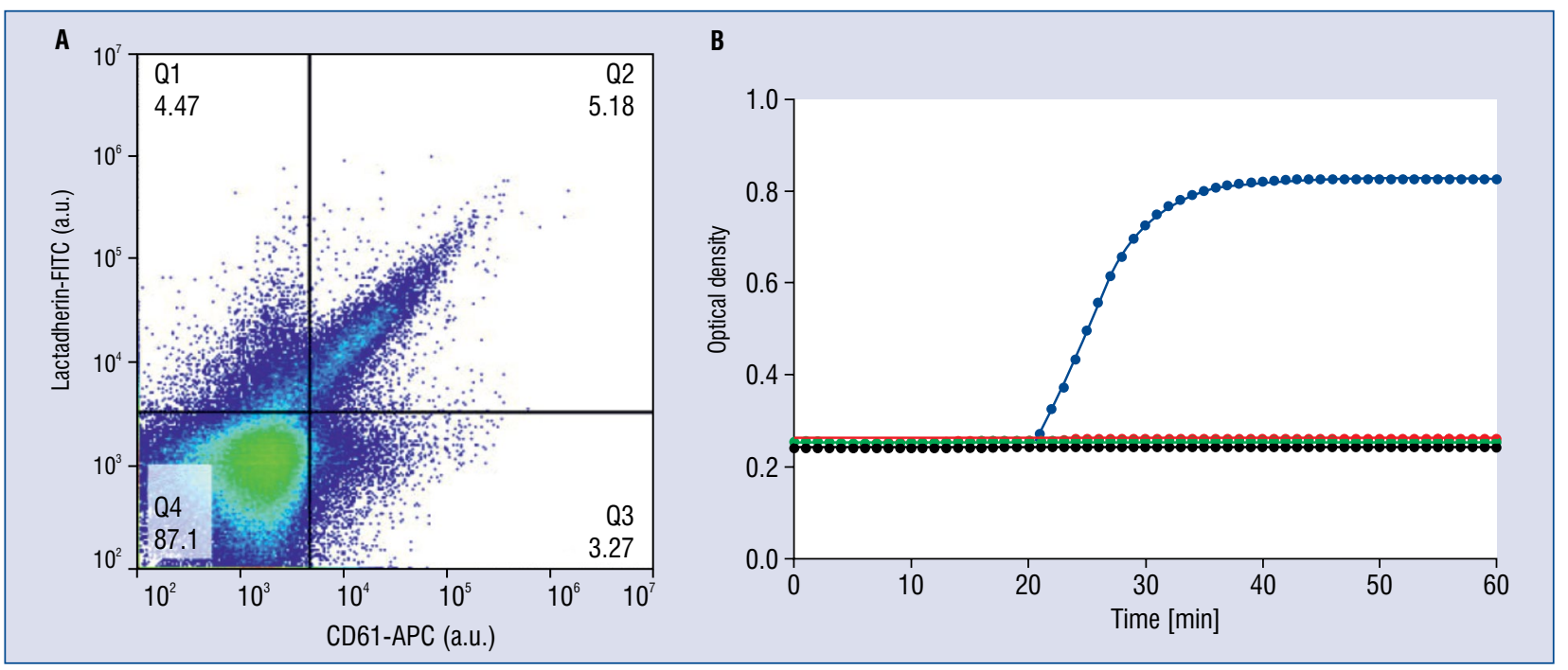

Figure 5. Proposed procoagulant activity of platelet extracellular vesicles (PEVs) in plasma. Fibrin generation test was performed in platelet-depleted, but PEVs-containing plasma stimulated with adenosine diphosphate (ADP). A part of PEVs expose phosphatidylserine $\left(\mathrm{CD} 1^{+} / \mathrm{PS}^{+}\right)(\mathrm{A})$. When the plasma clots, the optical density increases (B). ADP-stimulated plasma containing phosphatidylserine (PS)-exposing extracellular vesicles (EVs) did not clot (red). Upon the addition of recombinant human tissue factor (TF) to this plasma, clotting was triggered as measured by fibrin generation (blue). The extent of clotting was not affected by the presence of the P2Y1 and P2Y12 receptor antagonists (data not shown). Covering PS-exposing EVs with an excess of lactadherin, or inhibiting the TF-initiated extrinsic coagulation with an anti-factor VIla antibody, both inhibited plasma clotting (black and green, respectively). Hence, both PS-containing EVs and TF are indispensable for propagation of coagulation.

the release of distinct subpopulations of PEVs upon activation by ADP. The P2Y12 antagonist ticagrelor alone does not inhibit the release of PEVs-, suggesting that PEVs- may circulate in patients treated with ticagrelor and contribute to platelet homeostasis, for example by waste management [24]. On the contrary, ticagrelor decreases the release of PEVs+, that is PEVs exposing the proinflammatory P-selectin and procoagulant PS, suggesting that this subpopulation of PEVs may be decreased in patients treated with ticagrelor. In accordance, other P2Y12 receptor antagonists (clopidogrel, prasugrel and cangrelor) were shown to decrease the release of EVs from activated platelets as well [25-27], although the phenotype of the analysed PEVs was different than in the present study. Clopidogrel decreased the release of PEVs $\left(\mathrm{CD} 42 \mathrm{a}^{+}, \mathrm{PS}^{+}\right)$in response to thrombin receptoractivating peptide (TRAP) in a group of 12 patients with ACS [25]. Similarly, both prasugrel active metabolite and cangrelor decreases the collagen- and TRAP-induced release of PEV $\left(\mathrm{CD} 42 \mathrm{a}^{+}, \mathrm{PS}^{+}\right)$in a concentration-dependent manner in experimental conditions [26, 27].

Inhibition of P-selectin- and PS-exposing PEVs may. at least partly, explain the combined anti-thrombotic and anti-inflammatory benefits of the P2Y12 receptor antagonists. The potential proinflammatory and procoagulant effects of PEVs are presented in Figure 6. Although PS-exposing $\mathrm{EVs}$, such as those present in the plasma of healthy individuals, do not initiate coagulation in the absence of TF, PS-exposing EVs promote coagulation by providing a procoagulant surface. This procoagulant surface may be crucial in the ACS setting, where $\mathrm{TF}$ is exposed on a ruptured atherosclerotic plaque [28], and where PS-exposing EVs together with TF likely contribute to coronary thrombus formation. PEVs + may contribute to coagulation also indirectly by activating monocytes interaction of PEVs P-selectin with monocyte PSGL-1, leading to cytokine release and production of TF [9]. Thus, by decreasing the concentration of PEV+, ticagrelor likely decreases the proinflammatory/ procoagulant surface for interaction between $\mathrm{PEVs}$ and monocytes/clotting factors, respectively.

Regarding the recently proved inflammatory hypothesis of atherosclerosis [29] and the lack of consensus regarding the optimal duration of antiplatelet therapy with P2Y12 antagonists [4], determining the effect of P2Y12 antagonists on the release of PEVs helps to explain the mechanisms underlying decreases in inflammation/thrombosis for patients treated with P2Y12 antagonists. To determine the 


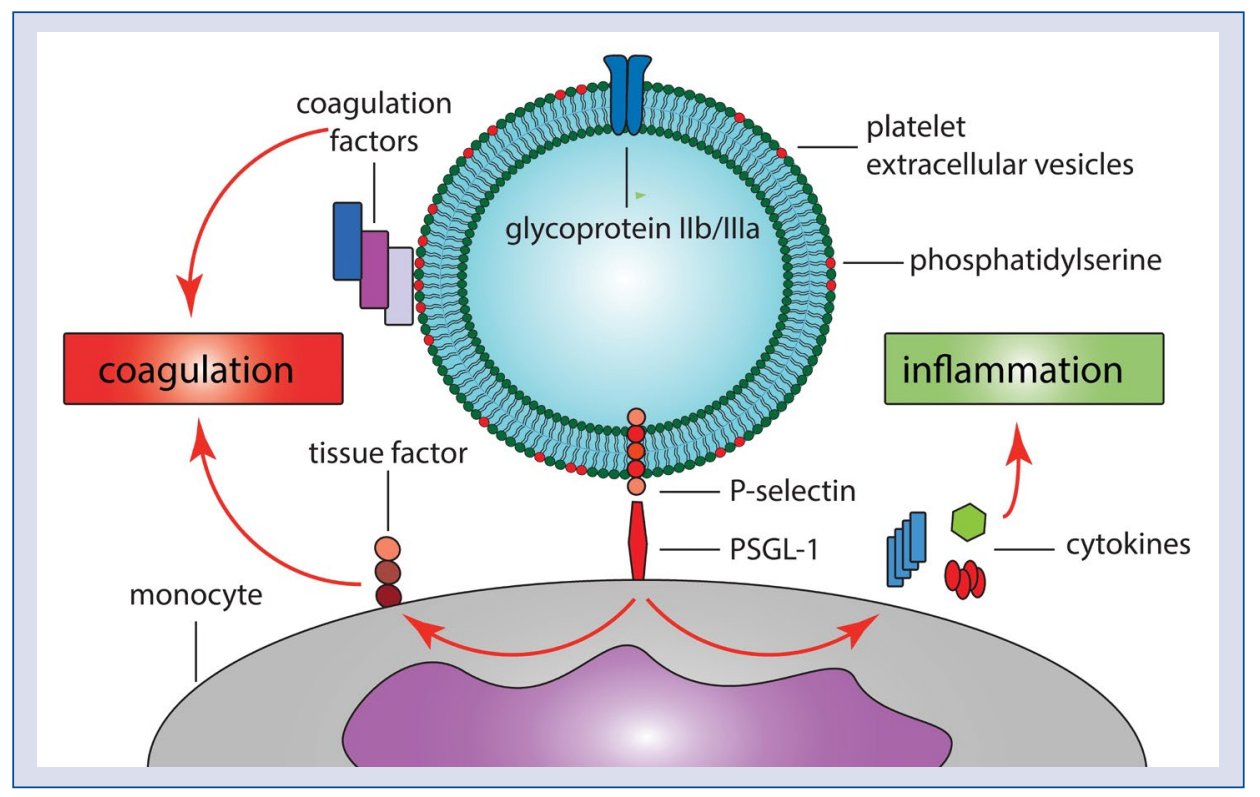

Figure 6. The potential proinflammatory and procoagulant effects of platelet extracellular vesicles (PEVs). PEVs contribute to coagulation both directly by providing procoagulant surface (phosphatidylserine) for the assembly of coagulation factors, and indirectly by binding of P-selectin to P-selectin glycoprotein ligand-1 (PSGL-1) on monocytes, leading to an exposure of tissue factor on monocytes. In addition, interaction between P-selectin and PSGL-1 contributes to inflammation by stimulating the release of pro-inflammatory cytokines from monocytes.

effects of the P2Y12 antagonists on the release of PEVs in a clinical setting, the AFFECT EV (Antiplatelet therapy effect on platelet extracellular vesicles, NCT02931045) study was launched [30]. AFFECT EV compares the effects of clopidogrel and ticagrelor on the release of PEVs in patients with ACS, with the hypothesis that more potent platelet inhibition is associated with larger decrease of PEV release.

\section{Limitations of the study}

First, the study was limited by the relatively small size of the study population, and therefore the presented results and conclusions should be considered as hypothesis-generating. Second, the decrease in the release of proinflammatory/procoagulant PEVs by ticagrelor was observed under experimental conditions only, and requires confirmation in a clinical setting. Third, the study identifies only one potential mechanism of the combined anti-thrombotic and anti-inflammatory benefits of the P2Y12 receptor antagonists - decrease of the release of PEVs. Since activated platelets release other proinflammatory/ /procoagulant molecules along with PEVs, it is likely that other mechanisms contribute to clinical benefits of the P2Y12 receptor antagonists as well.

\section{Conclusions}

Despite extensive research into the role and clinical applications of PEVs, the mechanisms regulating the release of PEVs as well as the effects of widely used drugs on the release of PEVs remain to be defined. The present results demonstrate that ADP-activated platelets released two distinct subpopulations of PEVs, which differ in their sensitivity to inhibition of $\mathrm{P} 2 \mathrm{Y} 1$ and $\mathrm{P} 2 \mathrm{Y} 12$ receptors. The release of (likely) proinflammatory and procoagulant PEVs is decreased by the P2Y12 antagonist ticagrelor, identifying a potential mechanism underlying attenuated inflammation and thrombosis in patients treated with P2Y12 antagonists. Further research is warranted to confirm this mechanism in vitro as well as in vivo.

\section{Acknowledgements}

We acknowledge funding from the Netherlands Organisation for Scientific Research - Domain Applied and Engineering Sciences (NWO-TTW), research program VENI 15924 (Edwin van der Pol).

Conflict of interest: None declared 


\section{References}

1. Linden MD, Jackson DE. Platelets: pleiotropic roles in atherogenesis and atherothrombosis. Int J Biochem Cell Biol. 2010; 42(11): 1762-1766, doi: 10.1016/j.biocel.2010.07.012, indexed in Pubmed: 20673808.

2. Hechler B, Gachet C. Purinergic receptors in thrombosis and inflammation. Arterioscler Thromb Vasc Biol. 2015; 35(11): 2307-2315, doi: 10.1161/ATVBAHA.115.303395, indexed in Pubmed: 26359511.

3. Yanachkov IB, Chang H, Yanachkova MI, et al. New highly active antiplatelet agents with dual specificity for platelet P2Y1 and P2Y12 adenosine diphosphate receptors. Eur J Med Chem. 2016; 107: 204-218, doi: 10.1016/j.ejmech.2015.10.055, indexed in Pubmed: 26588064.

4. Ibanez B, James S, Agewall S, et al. 2017 ESC Guidelines for the management of acute myocardial infarction in patients presenting with ST-segment elevation: The Task Force for the management of acute myocardial infarction in patients presenting with ST-segment elevation of the European Society of Cardiology (ESC). Eur Heart J. 2018; 39(2): 119-177, doi: 10.1093/eurheartj/ ehx393, indexed in Pubmed: 28886621.

5. van der Pol E, Böing AN, Harrison P, et al. Classification, functions, and clinical relevance of extracellular vesicles. Pharmacol Rev. 2012; 64(3): 676-705, doi: 10.1124/pr.112.005983, indexed in Pubmed: 22722893.

6. Rank A, Nieuwland R, Delker R, et al. Cellular origin of platelet-derived microparticles in vivo. Thromb Res. 2010; 126(4): e255-e259, doi: 10.1016/j.thromres.2010.07.012, indexed in Pubmed: 20696467.

7. Jurk K, Kehrel BE. Platelets: physiology and biochemistry. Semin Thromb Hemost. 2005; 31(4): 381-392, doi: 10.1055/s-2005916671, indexed in Pubmed: 16149014.

8. Vajen T, Mause SF, Koenen RR. Microvesicles from platelets: novel drivers of vascular inflammation. Thromb Haemost. 2015; 114(2): 228-236, doi: 10.1160/TH14-11-0962, indexed in Pubmed: 25994053.

9. Falati S, Liu Q, Gross P, et al. Accumulation of tissue factor into developing thrombi in vivo is dependent upon microparticle P-selectin glycoprotein ligand 1 and platelet P-selectin. J Exp Med. 2003; 197(11): 1585-1598, doi: 10.1084/jem.20021868, indexed in Pubmed: 12782720.

10. Heemskerk JWM, Mattheij NJA, Cosemans JM. Platelet-based coagulation: different populations, different functions. J Thromb Haemost. 2013; 11(1): 2-16, doi: 10.1111/jth.12045, indexed in Pubmed: 23106920.

11. Boulanger CM, Loyer X, Rautou PE, et al. Extracellular vesicles in coronary artery disease. Nat Rev Cardiol. 2017; 14(5): 259-272, doi: 10.1038/nrcardio.2017.7, indexed in Pubmed: 28150804.

12. Badimon L, Suades R, Fuentes E, et al. Role of Platelet-Derived Microvesicles As Crosstalk Mediators in Atherothrombosis and Future Pharmacology Targets: A Link between Inflammation, Atherosclerosis, and Thrombosis. Front Pharmacol. 2016; 7: 293, doi: 10.3389/fphar.2016.00293, indexed in Pubmed: 27630570.

13. Zaldivia MTK, McFadyen JD, Lim B, et al. Platelet-Derived Microvesicles in Cardiovascular Diseases. Front Cardiovasc Med. 2017; 4: 74, doi: 10.3389/fcvm.2017.00074, indexed in Pubmed: 29209618.

14. Gasecka A, Böing AN, Filipiak KJ, et al. Platelet extracellular vesicles as biomarkers for arterial thrombosis. Platelets. 2017; 28(3): 228-234, doi: 10.1080/09537104.2016.1254174, indexed in Pubmed: 27996341.

15. Gąsecka A, van der Pol E, Nieuwland R, et al. Extracellular vesicles in post-infarct ventricular remodelling. Kardiol Pol. 2018; 76(1): 69-76, doi: 10.5603/KP.a2017.0178, indexed in Pubmed: 28980299 .
16. Tomaniak M, Gąsecka A, Filipiak KJ. Cell-derived microvesicles in cardiovascular diseases and antiplatelet therapy monitoring - A lesson for future trials? Current evidence, recent progresses and perspectives of clinical application. Int J Cardiol. 2017; 226: 93-102, doi: 10.1016/j.ijcard.2016.10.007, indexed in Pubmed: 27792994

17. Morel O, Jesel L, Freyssinet JM, et al. Cellular mechanisms underlying the formation of circulating microparticles. Arterioscler Thromb Vasc Biol. 2011; 31(1): 15-26, doi: 10.1161/ATVBAHA.109.200956, indexed in Pubmed: 21160064.

18. Coumans FAW, Brisson AR, Buzas EI, et al. Methodological Guidelines to Study Extracellular Vesicles. Circ Res. 2017; 120(10): 1632-1648, doi: 10.1161/CIRCRESAHA.117.309417, indexed in Pubmed: 28495994.

19. van der Pol E, van Gemert MJC, Sturk A, et al. Single vs. swarm detection of microparticles and exosomes by flow cytometry. J Thromb Haemost. 2012; 10(5): 919-930, doi: 10.1111/j.15387836.2012.04683.x, indexed in Pubmed: 22394434.

20. van der Pol E, Coumans FAW, Sturk A, et al. Refractive index determination of nanoparticles in suspension using nanoparticle tracking analysis. Nano Lett. 2014; 14(11): 6195-6201, doi: 10.1021/n1503371p, indexed in Pubmed: 25256919.

21. Berckmans RJ, Sturk A, van Tienen LM, et al. Cell-derived vesicles exposing coagulant tissue factor in saliva. Blood. 2011; 117(11): 3172-3180, doi: 10.1182/blood-2010-06-290460, indexed in Pubmed: 21248061.

22. Arraud N, Linares R, Tan S, et al. Extracellular vesicles from blood plasma: determination of their morphology, size, phenotype and concentration. J Thromb Haemost. 2014; 12(5): 614-627, doi: 10.1111/jth.12554, indexed in Pubmed: 24618123.

23. Hechler B, Gachet C. Purinergic receptors in thrombosis and inflammation. Arterioscler Thromb Vasc Biol. 2015; 35(11): 2307-2315, doi: 10.1161/ATVBAHA.115.303395, indexed in Pubmed: 26359511.

24. Böing AN, Stap J, Hau CM, et al. Active caspase-3 is removed from cells by release of caspase-3-enriched vesicles. Biochim Biophys Acta. 2013; 1833(8): 1844-1852, doi: 10.1016/j.bbamcr.2013.03.013, indexed in Pubmed: 23531593.

25. Behan MWH, Fox SC, Heptinstall S, et al. Inhibitory effects of P2Y12 receptor antagonists on TRAP-induced platelet aggregation, procoagulant activity, microparticle formation and intracellular calcium responses in patients with acute coronary syndromes. Platelets. 2005; 16(2): 73-80, doi: 10.1080/09537100400005634, indexed in Pubmed: 15823862.

26. Judge HM, Buckland RJ, Sugidachi A, et al. The active metabolite of prasugrel effectively blocks the platelet P2Y12 receptor and inhibits procoagulant and pro-inflammatory platelet responses. Platelets. 2008; 19(2): 125-133, doi: 10.1080/09537100701694144, indexed in Pubmed: 18297550.

27. Judge HM, Buckland RJ, Holgate CE, et al. Glycoprotein IIb/ IIIa and P2Y12 receptor antagonists yield additive inhibition of platelet aggregation, granule secretion, soluble CD40L release and procoagulant responses. Platelets. 2005; 16(7): 398-407, doi: 10.1080/09537100500163226, indexed in Pubmed: 16236601 .

28. Tatsumi K, Mackman N. Tissue factor and atherothrombosis. J Atheroscler Thromb. 2015; 22(6): 543-549, doi: 10.5551/ jat.30940, indexed in Pubmed: 26016513.

29. Ridker PM, Everett BM, Thuren T, et al. CANTOS Trial Group. Antiinflammatory therapy with canakinumab for atherosclerotic disease. N Engl J Med. 2017; 377(12): 1119-1131, doi: 10.1056/ NEJMoa1707914, indexed in Pubmed: 28845751.

30. Medical University of Warsaw. Antiplatelet therapy effect on platelet extracellular vesicles (AFFECT EV). NLM Identifier: NCT02931045. https://clinicaltrials.gov/ct2/show/ NCT02931045 (Accessed: 18.02.2018). 